FORMATION Formation emploi

Revue française de sciences sociales

94 | avril-juin 2006

Immigration et marché du travail

\title{
Quelle professionnalisation dans le travail social pour les diplomés descendants de migrants?
}

What professionalising opportunities are available in the social field to graduates from immigrant families?

Welche Professionalisierung der Sozialen Arbeit für Akademiker mit

Migrationshintergrund?

\section{Marnia Belhadj}

\section{OpenEdition}

Journals

Édition électronique

URL : http://journals.openedition.org/formationemploi/2415

DOI : 10.4000/formationemploi.2415

ISSN : 2107-0946

Éditeur

La Documentation française

Édition imprimée

Date de publication : 1 avril 2006

Pagination : 73-91

ISSN : 0759-6340

Référence électronique

Marnia Belhadj, «Quelle professionnalisation dans le travail social pour les diplomés descendants de migrants? », Formation emploi [En ligne], 94 I avril-juin 2006, mis en ligne le 04 décembre 2009, consulté le 30 octobre 2020. URL : http://journals.openedition.org/formationemploi/2415 ; DOI : https://doi.org/10.4000/formationemploi.2415 


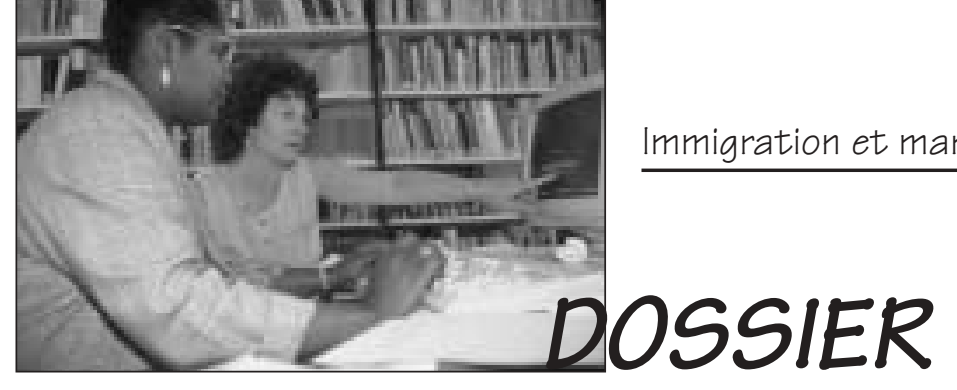

\section{Quelle professionnalisation dans le travail social pour les diplomés descendants de migrants?}

Par Marnia Belhadj*

Les difficultés économiques ont alourdi la tâche des travailleurs sociaux en dégradant la situation des populations les plus défavorisées, notamment immigrées. Dans ce contexte, quelle peut être la place des travailleurs sociaux eux-mêmes issus de l'immigration? Dans quelle mesure sont-ils l'objet d'une catégorisation particulière pour le recrutement et le déroulement de leur carrière?

La mise en place des politiques de la Ville et de développement social urbain ont profondément transformé les cadres d'exercice des professions sociales. Elles ont conduit les collectivités locales, (responsa-

\footnotetext{
${ }^{1}$ Cet article fait suite à une enquête réalisée dans le cadre du projet Balzan (responsable Dominique Schnapper) réunissant plusieurs autres chercheurs et professeurs d'université dont notamment Freddy Raphaël, professeur à l'université de Strasbourg 2, Serge Paugam, directeur de recherche au CNRS, Chantal BordesBenayoun, directrice de recherche au CNRS à l'université Toulouse le Mirail, Corinne Rostaing et Béate Collet, maîtres de conférences à l'université Lyon 2, Myriam Hachimi-Alaoui, chargée de recherche, Miléna Doytcheva, maître de conférence à l'université Lille 3 et Aline Soufflet chargée de recherche. Ce projet se propose de contribuer à développer la recherche sur l'intégration des sociétés contemporaines à partir de l'étude de différents milieux et différentes catégories de la population. L'objectif, à l'issue de ces travaux, est la mise en oeuvre d'une enquête quantitative nationale sur le lien de citoyenneté, qui sera réalisée sous la direction de Serge Paugam. Ce projet est financé par la fondation Balzan et le prix attribué à Dominique Schnapper en 2002.
}

* Marnia Belhadj est docteur en sociologie, chargée de recherche pour le programme Balzan à la Maison des sciences de l'Homme et enseignante au département de sociologie de l'université de Poitiers. Elle est responsable d'une recherche sur les processus de professionnalisation des descendants de migrants diplômés de l'enseignement supérieur dans le secteur social et au sein d'une grande entreprise de transports publics parisiens. Cette recherche est coordonnée par Dominique Schnapper (directeur d'études à l'EHESS - École des hautes études en sciences sociales - membre du conseil constitutionnel).

Ses domaines de recherche sont l'étude des parcours de mobilité sociale des enfants de migrants, la famille, les relations intergénérationnelles, l'éducation, le travail social, les parcours d'insertion, les trajectoires scolaires, professionnelles, familiales, résidentielles, la citoyenneté, les politiques de la ville. 
Elle a notamment publié : Belhadi M. (2006), La conquête de l'autonomie. Histoire de Françaises descendantes de migrants algériens, Éditions de l'Atelier, Paris. Belhadj. M. (2004), "Das républikanische Intégrationsmodel auf dem Prüfstand", in Bizeul Yves, Integration von Migranten, Französische und deutsche Konzepte im Vergleich, Deutsher Universitäts-Verlag, pp. 33-44. Balhadi M. (2003), "Choix du conjoint et stratégies matrimoniales d'un groupe de femmes françaises d'origine algérienne», Revue Européenne des Migrations Internationales (REMI), 15, avril-mai, pp. 195-222.

bles des Missions locales, élus municipaux chargés de l'emploi, ANPE...) chargées de mettre en place les dispositifs d'aide à l'insertion et d'aménagement des quartiers à identifier de nouvelles catégories d'acteurs susceptibles de relever le défi de l'insertion. Le travail de ces nouveaux acteurs s'organise principalement autour de l'aide, de l'intervention, de la prévention et de l'accompagnement des populations les plus fragiles et les plus vulnérables habitant un secteur ou un quartier défini socialement et territorialement. Ces fonctions mobilisent également des compétences ou des savoirs particuliers, notamment la connaissance du milieu et de l'environnement social et territorial dans lequel le dispositif est mis en place. Beaucoup de fils et filles de migrants diplômés de l'enseignement supérieur, issus ou non de quartiers populaires, et désireux de faire reconnaître leurs compétences, ont trouvé là un moyen de concilier à la fois leur formation universitaire et leurs aspirations professionnelles. Quelle place occupent ces nouveaux professionnels dans le champ du travail social et quel rôle jouent-ils dans le processus de renouvellement des rapports sociaux? Il s'agit de comprendre dans quelle mesure leurs projets professionnels se confrontent aux logiques et aux besoins institutionnels qui privilégient certaines compétences et connaissances particulières, pour aboutir, à terme, à un processus de catégorisation, voire d'ethnicisation. Quelles en seront alors les conséquences et les enjeux tant pour le secteur social en termes de renouvellement des cadres légaux d'exercice de ces professions que pour les professionnels eux-mêmes en termes de professionnalisation, de valorisation des compétences et de perspectives professionnelles ?
Nous tenterons d'apporter quelques éléments de réponse à ces questions à partir des résultats d'une enquête réalisée dans le secteur du développement social et urbain auprès de professionnels du travail social, descendants de migrants, et de responsables chargés de recrutement (voir encadré 1). La confrontation entre des logiques institutionnelles (privilégiant certaines compétences endogènes) et les projets des professionnels permet de mieux comprendre les motivations des uns et des autres et les processus à l'œuvre dans ce secteur d'activité.

Pour ce faire, il nous faut tout d'abord revenir sur les parcours de ces nouveaux professionnels du travail social. Nous observerons ainsi les similitudes et les disparités avec les parcours des travailleurs sociaux issus des générations précédentes et appartenant aux couches populaires, notamment en termes de mobilité sociale et de professionnalisation. À partir des entretiens réalisés avec les responsables de recrutement, nous étudierons ensuite les mécanismes qui conduisent à une catégorisation professionnelle. Dans quelle mesure les professionnels descendants de migrants s'inscrivent-ils dans cette catégorisation et quel rôle jouent-ils à l'intérieur du secteur social ? C'est ce que nous avons tenté de savoir à l'issue de cette étude à partir de leur rapport au travail et de leurs objectifs professionnels.

\section{UNE PROFESSIONNALISATION NÉGOCIÉE}

L'enquête a été réalisée en région parisienne avec une vingtaine de fils et de filles de migrants travaillant dans le secteur du développement social des quartiers, tous diplômés de l'enseignement supérieur (encadré 1). Elle révèle notamment que les parcours de ces professionnels ne sont pas très différents de ceux qu'ont pu connaître leurs aînés issus des milieux populaires. Il existe des traits communs à toutes ces générations qui ont été les premières dans leurs milieux à accéder aux diplômes et aux emplois qualifiés. Leurs parcours professionnels est la résultante d'une histoire familiale, sociale et personnelle qui les a conduits notamment à s'orienter vers des métiers et des domaines dans lesquels ils ont le sentiment qu'ils seront utiles et qu'ils auront un rôle actif à jouer. Cette histoire nous informe sur leurs cheminements et relativise certains traits que l'on voudrait spécifiques 
à telle ou telle catégorie, alors qu'ils sont la plupart du temps partagés par une majorité d'individus appartenant aux mêmes milieux. Sauf que, dans le cas des enfants de migrants, l'inscription dans l'espace social et spatial est telle qu'elle constituera pour certains responsables de recrutement un élément déterminant dans leur recrutement et leur participation à l'action et à l'intervention sociale. Dès lors,

\section{Encadré 1 \\ Méthodologie de l'enquête}

L'enquête par entretiens biographiques a été réalisée dans les quartiers Nord de deux communes de la région parisienne, auprès d'une trentaine de personnes travaillant dans des structures d'aide à l'insertion professionnelle (Mission locale, Maison de l'emploi, Régie de quartier, PAIO - Permanence d'Aide, d'Insertion et d'Orientation) dont dix conseillers à l'insertion professionnelle (travaillant essentiellement dans les Missions locales) ; une chargée d'accueil (Maison de l'emploi et Mission locale) ; quatre chargés de formation; six chefs de projet, responsables de Régie de quartier (contrat de ville) ; cinq responsables de structure et de recrutement dont : trois directeurs de Mission locale ; un responsable de la Maison de l'emploi, un responsable de PAIO.

Profil des enquêtés des quatre premières professions :

Douze hommes et neuf femmes, âgés de 29 à 42 ans, de niveau bac +2 à bac +5 (DEA - Diplôme d'études approfondies - DESS - Diplôme d'études supérieures spécialisées - , sciences humaines (sociologie, anthropologie, sciences politiques), diplôme supérieur d'expertise comptable, expérience dans le travail social (animation en centre aéré, maison des jeunes, centre social...). Tous sont nés ou ont grandi en France et ont vécu dans des quartiers populaires. Certains ont connu les quartiers dits sensibles comme le Val-Fourré (Mantes la Jolie) ou la cité des quatre mille à la Courneuve. Leurs parents, ouvriers pour la plupart, sont d'origine algérienne ou marocaine et sont arrivés en France à la fin des années 50.

Les responsables de recrutement

Quatre hommes et une femme âgés de 36 à 52 ans. Trois d'entre eux ont une formation classique en travail social (assistant en travail social, éducateur ou formateur) et possèdent un diplôme d'État. Ils sont enfants d'ouvriers ou d'employés; deux ont effectué des études supérieures en sciences humaines et sont titulaires d'un doctorat ou d'un DEA. Ces derniers (un homme et une femme) sont issus de milieux sociaux plutôt favorisés et sont venus en France dans les années 80 pour poursuivre des études supérieures.

Les entretiens individuels d'environ 90 minutes à deux heures ont eu lieu pour la plupart sur le lieu de travail, dans les bureaux des professionnels et des chargés de recrutement. La plupart des entretiens n'a nécessité qu'une séance.

Les thèmes abordés avec les enquêtés (la trajectoire des familles, l'éducation, la scolarité, l'insertion professionnelle, les activités sociales, associatives et politiques, les loisirs, leur position à l'intérieur des quartiers...) permettaient de reconstituer leurs différents parcours ainsi que les systèmes de valeurs et de représentations. Les entretiens biographiques ont été complétés par des observations directes sur le terrain. Cela a consisté à être présente, avec l'accord des responsables de structure, dans les salles collectives où se rassemblaient les jeunes des Missions locales pour utiliser l'outil informatique ou consulter des brochures professionnelles, encadrés en cela par un formateur ou un conseiller en insertion. L'intérêt a surtout porté sur les relations entre les jeunes et les conseillers dans le cadre de cet encadrement. Ma présence (une fois par semaine durant deux mois) ne semble pas avoir affecté les activités, dans la mesure où je passais très souvent pour une personne à la recherche d'un emploi venant consulter les brochures et utiliser Internet. 
comment s'opère la continuité des parcours des professionnels descendants de migrants avec ceux qu'ont pu connaître les autres professionnels issus de couches populaires ? À quel moment survient la rupture entre ces deux parcours?

\section{Le travail social : une voie de mobilité sociale pour les enfants d'ouvriers}

L'appartenance sociale et familiale a une forte incidence sur les trajectoires scolaires et professionnelles des individus. Beaucoup de travailleurs sociaux ayant pris leurs fonctions dans les années 60 et 70 sont issus de familles ouvrières. Cet élément n'est pas anodin lorsque l'on sait que la grande majorité des descendants de migrants travaillant dans le secteur social sont eux aussi d'origine ouvrière. En effet, les carrières du travail social ont longtemps constitué, avec celles de l'enseignement, une voie permettant de concilier poursuite d'études supérieures, même courtes, et accès rapide à l'emploi. C'est aussi pour cette raison que le travail social a longtemps représenté un réservoir d'emplois pour les enfants d'ouvriers accédant à l'enseignement supérieur. Aujourd'hui, ce domaine représente aussi un débouché pour les filières en sciences humaines, par le biais notamment de parcours spécifiques dans le cadre des nouvelles réformes. Celles-ci ont mieux défini certains parcours permettant par exemple aux étudiants d'opter pour une licence professionnelle en intervention sociale option «accompagnement et insertion », qui pourra être complétée par un master professionnel.

Le travail social constitue donc une voie de mobilité sociale privilégiée pour les enfants de milieux ouvriers. En ce sens, devenir assistant en service social ou éducateur peut être considéré dans les milieux populaires comme une forme d'ascension sociale au même titre que chef de projet ou conseiller en insertion. Dès lors, il existe une continuité dans les parcours professionnels qui permet à la fois un accès rapide à l'emploi et une mobilité sociale pour les enfants d'ouvriers, qu'ils soient ou non descendants de migrants. Ce secteur semble plus accessible aux descendants de migrants diplômés de l'enseignement supérieur que d'autres secteurs d'activité, comme nous l'a confié une responsable de projet. L'ANPE lui a proposé d'emblée un poste dans une structure d'in- sertion professionnelle nouvellement créée dans une commune de la région parisienne :

"Que l'on soit ou non formés pour ça, on nous propose presque toujours des emplois sociaux lorsque l'on s'adresse à l'ANPE. Moi j'ai une licence d'AES, a priori je ne suis pas formée pour travailler dans une PAIO et quand je me suis présentée à l'ANPE, on m'a dit, il y a une Permanence qui va être créée dans tel quartier et ils ont besoin d'un responsable, si ça vous intéresse vous pouvez vous y rendre. Quand j'y suis allée, j'ai eu un entretien avec un responsable du recrutement et deux jours après j'étais prise. Voilà comment j'ai atterri ici. Mais faut pas se voiler la face, c'est plus facile de travailler ici qu'à la banque ou dans un cabinet d'avocats..." (Samia, 31 ans responsable de PAIO, licence d'AES administration économique et sociale).

Ainsi, le secteur social et les nouveaux emplois créés dans le cadre des politiques de la Ville seraient d'un accès plus facile que les emplois du secteur bancaire ou libéral. Ils représentent pour certains professionnels un palliatif aux difficultés rencontrées dans d'autres secteurs. C'est du moins ce que suggèrent certains propos de personnes interviewées qui semblent s'être dirigées vers le travail social par défaut, soit parce qu'elles ont été refusées ailleurs, soit parce que la sélection sévère de certains concours de la fonction publique les a découragées. En l'absence d'éléments de comparaison dans ce domaine, il est difficile d'affirmer que ces comportements constituent une réponse à certaines pratiques discriminatoires pouvant s'exercer de manière plus massive à l'égard des descendants de migrants. Cependant, on peut supposer qu'objectivement ces pratiques existent et qu'elles pourraient jouer un rôle dans les parcours professionnels des diplômés de l'enseignement supérieur descendants de migrants (Philippe Bataille, 1997).

La continuité des parcours de ces nouveaux professionnels avec ceux des autres travailleurs sociaux s'observe également dans la familiarité avec le domaine social. Les familles ouvrières de conditions modestes constituent très souvent la majorité du public pris en charge par les travailleurs sociaux. Ces familles font souvent l'objet d'un encadrement social qui les conduit à côtoyer fréquemment ces professionnels. Elles connaissent donc certainement mieux ces professions qui font partie de leur environnement quotidien que d'autres professions plus éloignées. 
Dès lors, cette proximité ou cette familiarité peut influer sur les orientations professionnelles non pas parce que ce domaine a pu faire naître des vocations mais parce que certains aspirent à en modifier les cadres et les pratiques :

" J'ai toujours voulu travailler dans le social, mais je ne voulais pas devenir éducateur ou assistant social. $J$ 'en ai trop vu dans mon enfance et je n'aimais pas trop la manière dont ils s'occupaient des familles maghrébines. Les assistantes sociales que je connaissais étaient de vraies fonctionnaires et elles s'occupaient plus de vérifier si ces familles ne percevaient pas des allocations auxquelles elles n'avaient pas droit que de leur procurer des aides. C'est pour cette raison aussi que les familles craignaient les assistantes sociales, elles avaient peur qu'elles leur suppriment les aides auxquelles elles avaient droit. Elles n'avaient aucune connaissance de ces populations et aucune proximité avec elles. Moi j'ai pas les mêmes rapports avec ces familles, ni le même regard, je suis plus proche d'elles et elles me font confiance. C'est différent, et c'est aussi pour ça que j'ai voulu travailler dans le social, pour être plus proches des gens... (Farid, 28 ans, chef de projet dans le cadre du contrat de Ville, BTS - brevet de technicien supérieur - d'action sociale et diplôme d'animateur socioculturel).

Au-delà des motivations qui conduisent les individus à s'orienter vers telle ou telle profession, leur trajectoire est aussi l'aboutissement d'un cheminement qui les inscrit à l'intérieur d'un espace social et d'un champ des possibles qui leur est objectivement ouvert et accessible.

\section{L'inscription dans l'espace social}

Dans les deux cas, la proximité, voire la solidarité avec le public auquel ces nouveaux professionnels sont confrontés dans le cadre de leur travail, peut représenter un élément déterminant dans leur cheminement professionnel. Les membres de cette profession partagent parfois certaines valeurs familiales, sociales ou politiques. Ainsi l'expérience militante et le rejet des inégalités sociales sous toutes ses formes ont souvent conduit les travailleurs sociaux des années 70 à s'engager dans les mouvements confessionnels (catholicisme social, charité...) (voir encadré 2). De manière analogue, un certain nombre de professionnels de l'intervention sociale, descen- dants de migrants, sont issus de mouvements associatifs des années 80 (centrés autour de la citoyenneté et de l'animation des quartiers). Ceux qui considèrent leur travail comme un engagement ( $c f$. le chapitre sur le rapport au travail) et comme une forme de prolongement de leurs activités militantes s'inscrivent dans cette tradition du travail social et dans cette continuité. Leur inscription dans l'espace social constitue une dimension importante de leur orientation professionnelle. Ce n'est pas tant leur formation qui les a préparés à devenir des professionnels dans le cadre de l'intervention sociale que leur parcours social, militant et associatif :

"Je n'ai pas fait d'études spécifiques dans le social. J'ai un diplome d'expert-comptable. J'ai travaillé dans un cabinet d'expert comptable un moment puis j'ai voulu faire autre chose. Car j'ai beaucoup milité étant plus jeune dans les associations. C'est plus par ma connaissance du terrain et par mes activités associatives que je suis arrivé là que par ma formation. C'est vrai que connaître la comptabilité ça aide pour diriger une structure mais bon ce n'est pas suffisant, il faut aussi aimer le contact avec les gens et les accompagner dans leurs parcours sociaux et professionnels et tout ça je l'ai souvent appris à travers mes activités associatives, par mon engagement sur le terrain » (Karim, 36 ans, directeur d'une Régie de quartier, diplôme d'expertise comptable).

Une partie des professionnels rencontrés a milité au sein de mouvements associatifs ou politiques. Certains ont été éducateurs ou animateurs socioculturels avant d'occuper des postes dans le champ de l'intervention sociale et du développement social et urbain. Leur parcours professionnel résulte donc aussi d'une expérience militante au cours de laquelle ils ont tissé des liens avec les élus locaux et constitué des réseaux qui ont certainement été déterminants dans leurs choix professionnels. Ces expériences sont partagées par un grand nombre de travailleurs sociaux. Elles n'avaient aucun caractère stigmatisant pour les travailleurs sociaux issues des générations précédentes, alors qu'elles jettent parfois le discrédit et le soupçon sur les professionnels sociaux descendants de migrants. Le cumul de plusieurs fonctions ou statuts comme celui de militant, de responsable associatif, d'habitant de quartier et de professionnel peut en effet conduire certains collègues appartenant aux métiers traditionnels du travail social à douter de 


\section{Encadré 2}

\section{Le Travail social : tradition, mutations et nouvelles logiques de professionnalisation}

Au début des années 70, I'appellation de travailleur social (regroupant le corps des assistants en service social, des éducateurs spécialisés et des animateurs socioculturels) s'est imposée d'un point de vue administratif, articulant ainsi le champ du travail aux différents métiers qui le composent U. lon, J.P Tricart, pp. 8-10). Le travailleur social est alors défini comme unique et polyvalent et le travail social est délimité, mis sous tutelle de l'administration de l'Action sociale qui lui reconnaît une légitimité par une formation supérieure et des diplômes.

Depuis la fin des années 70 , le secteur social a connu de multiples mutations, dues principalement à la détérioration des conditions socio-économiques et à l'accroissement des phénomènes d'exclusion et de précarité. De nouvelles politiques sont alors mises en place avec comme objectif essentiel de rétablir le lien social entre les individus les plus fragilisés et le reste de la société. Le défi de l'insertion devient dès lors un enjeu des référentiels de l'intervention sociale, nécessitant de nouvelles formes de médiation. Celles-ci sont basées sur la contractualisation et le suivi individualisé dans le cadre notamment du RMI (Revenu minimum d'insertion) et de la mise en place du contrat d'insertion. Ce dispositif a institué un partenariat entre les différents acteurs du champ social et les collectivités locales lélus locaux, municipalité, ANPE - Agence nationale pour l'emploi, ...) et modifié les approches des publics et les cadres de l'exercice professionnel (U.-N Chopart, 2000). Les « usagers » des travailleurs sociaux ne sont plus désormais perçus comme des assistés mais comme des acteurs qu'il faut accompagner et mobiliser autour d'un projet de vie et de trajectoire. II ne s'agit pas seulement de résoudre les problèmes mais de développer chez les personnes, désormais actrices, la capacité à se responsabiliser, à se mobiliser et à se mouvoir dans un réseau de partenaires qui pourront non plus seulement les aider mais aussi les accompagner dans leur parcours. À cet égard, la signature d'un contrat, au moins moral, comme celui qui est signé dans le cadre du RMI, peut signifier un engagement de la personne en tant qu'acteur (P. Billion, 200 1). Les cadres légaux et statutaires d'exercice de ces professions sont remis en cause par la politique de décentralisation. L'État notamment n'est plus le principal employeur des travailleurs sociaux. La tutelle du travail social se fragmente entre de multiples employeurs (le Département tout d'abord,) même si l'administration des Affaires sociales conserve la tutelle de la formation. On assiste notamment à une redéfinition et à une diversification des stratégies des organismes employeurs et des milieux professionnels. De nouvelles qualifications émergent, et ce qui faisait autrefois l'apanage de certaines professions sociales devient un principe d'action et d'intervention pour de nombreux professionnels.

L'appareil de formation se trouve lui aussi affecté par cette recomposition. Alors qu'il avait constitué un important levier dans les processus de professionnalisation (diplômes nationaux, monopoles professionnels, etc.) il peine à exercer un rôle d'homogénéisation. De nouvelles logiques de professionnalisation apparaissent, malgré le maintien de principe d'une tutelle de l'État (U.-N Chopart, 1994). De nouvelles initiatives internes ou externes aux milieux traditionnels de l'action sociale émergent, en particulier dans les milieux universitaires, avec la mise en place de nouveaux diplômes: "préqualification » aux fonctions sociales, DESS (Diplôme d'études supérieures spécialisées) de développement social ou urbain et, tout récemment, les licences professionnelles et les masters en sciences humaines et sociales mention "intervention sociale », qui se substituent de plus en plus aux DESS. La technicité et les compétences des nouveaux diplômés peuvent être contestées par d'autres professionnels avec lesquels ils sont en partenariat et qui leur reprochent notamment leur méconnaissance de l'économie, de la formation professionnelle et des systèmes d'emploi et des programmes d'insertion (I.-N Chopart, 2000). Ces phénomènes sont une des conséquences directes du développement des politiques de la Ville qui font du territoire et du quartier les pôles centraux de l'intervention et de l'action du travail social. L'objectif de 
ces interventions est double : l'insertion et la formation professionnelle pour l'accès à un emploi ; la vie urbaine à travers notamment l'accès au logement. Deux figures principales émergent de ces nouveaux dispositifs : les formateurs issus de la formation professionnelle continue, qui sont aussi les acteurs de base de ces nouvelles politiques, et les chefs de projet qui en sont les animateurs. Ces nouvelles professions, à mi-chemin entre le politique et le technique, cohabitent avec les métiers traditionnels sans pour autant établir de collaboration étroite. Certains voient une opposition radicale entre les nouvelles et les anciennes professions. Ils considèrent que les nouvelles politiques publiques opèrent une véritable rupture de sens et de contenu du travail qui remet en cause l'image des métiers traditionnels. D'autres, au contraire, minorent les répercussions de ces transformations sociales et professionnelles sur les conditions d'exercice de ces métiers.

l'impartialité de ces nouveaux professionnels et de leur capacité à se positionner à l'égard de leurs pairs et de leurs congénères (P. Billion, 2001). Cela soulève la question de la place et du rôle de ces professionnels au sein du champ du travail social mais aussi des motifs qui les ont incités à choisir ce domaine d'activité alors que leur formation initiale ne les prépare pas forcément à travailler dans ce secteur d'activité.

\section{Différence dans la formation et la professionnalisation}

Au-delà de ces similitudes, les différences entre les parcours de ces nouveaux professionnels et les autres travailleurs sociaux issus de mêmes milieux sociaux sont nombreuses. Elles mettent en évidence la singularité des nouveaux professionnels descendants de migrants. À commencer par la formation. Au sein des générations précédentes, beaucoup de travailleurs sociaux ont opté pour des formations spécialisées et professionnalisantes dispensées par les instituts et les écoles de formation des travailleurs sociaux. Il y ont acquis à la fois des savoirs théoriques en sciences sociales et des savoirs professionnels sous forme de stages. Les enfants d'ouvriers, descendants de migrants, quant à eux, sont pour la plupart entrés à l'université pour suivre une formation générale en sciences humaines. C'est essentiellement par la pratique professionnelle qu'ils deviendront des professionnels de l'intervention sociale.

Le niveau d'études de ces nouveaux professionnels est souvent plus élevé que celui des assistants en service social ou des éducateurs. Les chefs de projets, les conseillers en insertion et les responsa- bles de Régies de quartier possèdent en général un niveau d'études supérieur à bac +3 . Toutes les personnes interviewées dans le cadre de notre enquête ont une formation universitaire en sciences humaines (sociologie, anthropologie, sciences économiques ou sciences politiques) ou ont intégré une école supérieure d'expertise comptable. Elles sont toutes diplômées d'une maîtrise, d'un DEA ou d'un DESS et cela constitue très souvent une des conditions sine qua non de leur recrutement. Cette formation universitaire répond notamment aux attentes des responsables de recrutement que nous avons rencontrés. Tous insistent sur la nécessité, pour les professionnels du travail social, de posséder une formation générale de haut niveau, conjuguée à une expérience confirmée au sein d'un secteur du travail social, de préférence dans le domaine au sein duquel ils exerceront leurs fonctions. Ainsi, la plupart des conseillers recrutés avaient déjà travaillé dans des centres aérés ou culturels ou encore dans des centres sociaux comme animateurs ou chargés d'accueil. L'expérience acquise au cours de leurs études, ou à l'issue de celles-ci, a constitué un premier contact avec le travail social

D’une manière générale, les professions du travail social mobilisent des compétences scolaires et professionnelles diverses et inégales selon les fonctions et les publics. Si aujourd'hui l'appareil de formation connaît une recomposition et de nouvelles logiques de professionnalisation (voir encadré 1), les métiers déjà anciens sont mieux structurés, mieux reconnus et certifiés par des formations et des diplômes que la grande majorité des nouveaux emplois sociaux. Ceux-ci ne donnent lieu généralement à aucune formation spécifique (voir encadré 2). Les professionnels proviennent d'horizons divers 
(anciens militants et bénévoles, diplômés de l'enseignement supérieur, secteur associatif...). Leur formation ne les prépare pas davantage aux fonctions qui leur seront confiées. Pour certains postes liés à l'impératif d'insertion, c'est le modèle de contractualisation et de projet qui domine. Il ne s'agit plus de gérer les marginalités sur un mode psycho-éducatif mais d'élaborer, en concertation avec d'autres professionnels, un programme contractuel de l'insertion sociale, fondé sur les ressources locales et répondant au droit des personnes (J. Danzelot, 1984 et $c f$. encadré 2). C'est à cet impératif que doivent répondre les missions engagées par les travailleurs sociaux en général et par les nouveaux professionnels de l'intervention sociale en particulier. L'approche individualisée des parcours et des publics se conjugue avec le principe de partenariat entre les travailleurs sociaux, mais aussi entre ces derniers et les élus locaux qui devront travailler de concert dans le cadre de ce programme d'insertion (voir encadré 2).

L'accès à l'emploi devient donc la priorité des actions ; la problématique de l'insertion se substitue progressivement aux anciens mots d'ordre de l'action sociale qui visaient surtout la sphère familiale et éducative. Dans le cas des intervenants sociaux, la formation initiale est souvent éloignée de ce référentiel, et c'est davantage au cours de l'exercice d'une activité au sein du travail social, puis dans le cadre de leur travail, qu'ils vont faire l'apprentissage théorique et pratique des dispositifs d'insertion.

Ainsi, la formation initiale sera accompagnée d'une expérience concrète du travail social nécessitant d'une part, la connaissance de dispositifs d'insertion et d'autre part, celle du terrain et du public concerné par ces dispositifs. Ces conditions représentent donc le cadre de leur recrutement et du processus de professionnalisation qui se déroulera tout au long de leur parcours professionnel. Mais ce processus de professionnalisation résulte aussi d'une négociation des compétences et du statut entre les différents acteurs en présence (professionnels, responsables de recrutement et employeur). Dans ce secteur du travail social, aux logiques professionnelles complexes et multiples, l'articulation du travail à la série d'actions accomplies par les différents intervenants passe non pas par les postes formels bien définis mais par de véritables associations de compétences qui dépassent le seul cadre du secteur d'activité (D. Vrancken, 1994). Il s'agit moins de savoir si le métier exercé répond au critère de la profession au sens strict du terme que de comprendre dans quelle mesure les individus caractérisés par un métier s'efforcent de le transformer en profession et comment ils s'attachent eux-mêmes à en devenir les titulaires (Hugues, 1958). C'est sans doute aussi dans cette appropriation de la profession que les intervenants sociaux s'efforcent de faire reconnaitre leurs compétences et leurs savoirs. Bien que reconnues lors du recrutement, les compétences ne sont pas figées et peuvent être redéfinies au cours du parcours professionnel selon les évolutions du cadre légal des professions et des pratiques professionnelles. En ce sens, la professionnalisation repose aussi sur la capacité d'adaptation des individus et sur leur aptitude à valoriser leurs savoirs et savoir-faire afin d'être reconnus non seulement comme une force de travail mais comme acteurs au sein d'une profession qu'ils se sont appropriée et dont ils sont désormais titulaires.

Plus concrètement, le processus de professionnalisation des descendants de migrants enquêtés répond à une double logique : celle émanant des recruteurs qui privilégient des critères à la fois objectifs et endogènes et celle des recrutés qui s'efforcent de faire reconnaître à la fois les compétences et les expériences acquises au cours de leurs parcours scolaires et professionnels et le rôle qu'ils jouent au sein du travail social.

\section{LE RECRUTEMENT INDUIT UNE CATÉGORISATION}

La volonté des politiques de la Ville de corriger les inégalités observées dans les quartiers les plus déshérités aboutit à une catégorisation sociale et territoriale des habitants. En raison du regroupement massif des populations migrantes dans ces quartiers, les critères sociaux se retrouvent en réalité étroitement liés à des catégories que l'on pourrait qualifier d' « ethniques » dans d'autres pays (Schnapper, 2002). Au sens large, l' « ethnique » désigne, selon la formulation de Max Weber (1995), la croyance en une ascendance commune fondée sur la religion, l'« origine», la « race» ou la «culture». On parlera alors plus souvent d'ethnicité qui constitue un terme-clé de la littérature contemporaine aux États-Unis, notamment pour désigner, à l'intérieur de la société américaine, la force d'appartenance à des groupes particuliers (les Noirs, les Juifs, les Italos-Américains, les IrlandaisAméricains...) (Martinello, 1995). En étudiant les 
phénomènes identitaires en termes de rapports sociaux, d'inégalité et de pouvoir (Martinello, 1995), la plupart des travaux sur l'ethnicité soulignent le caractère socialement construit des identités particulières.

Le modèle républicain français, fondé sur l'égalité et les principes universels et émancipateurs de la citoyenneté, s'est officiellement toujours refusé à reconnaître les appartenances particulières. On peut retrouver dans les politiques de la Ville, des politiques indirectes de gestion de l'ethnicité (Miléna Doecheva, 2005), notamment par le biais du prisme que représentent les «banlieues défavorisées ». Dans ce cadre, l'objectif consiste plus à prendre en charge des diversités culturelles qu'à reconnaître des droits et des avantages spécifiques. La prise en compte de la diversité culturelle constitue un référentiel du travail social et des pratiques des intervenants sociaux. On retrouve également cette préoccupation dans les pratiques de recrutement des nouveaux professionnels du social. Les actions menées dans le cadre du développement social des quartiers s'adressent prioritairement à leurs habitants, souvent issus de populations migrantes; dès lors, l'appartenance (réelle ou supposée) ou la proximité avec ces populations constituera un critère important dans le recrutement des professionnels. De ce point de vue, ces pratiques de recrutement peuvent s'accompagner d'un processus d'ethnicisation des rapports sociaux ; il s'agit d'identifier une nouvelle catégorie de professionnels définis socialement et territorialement mais aussi ethniquement à partir de dispositions liées à leur parcours, leurs origines sociales, familiales ou culturelles, à leur socialisation et leurs expériences, pour qu'ils exercent leur mission auprès d'une population partageant a priori les mêmes caractéristiques. Dans les faits, les deux critères couplés de l'expérience et de l'appartenance conduisent à choisir essentiellement des descendants de migrants.

Ces phénomènes induisent donc une catégorisation. Elle consiste à cibler de nouvelles catégories de travailleurs sociaux à la fois à partir de critères scolaires et professionnels et de critères endogènes liés à leur appartenance sociale, familiale et territoriale (Billion, 2004). Comment s'opère cette catégorisation et dans quelle mesure peut-elle aboutir à un processus d'ethnicisation? C'est ce que nous tentons de comprendre grâce à l'étude des pratiques des responsables de recrutement que nous avons interviewés.

\section{Une «présomption de compétence »}

Beaucoup de responsables que nous avons rencontrés, chargés de recruter les conseillers en insertion et les chefs de projets, estiment que les candidatures des descendants de migrants sont objectivement traitées de la même manière que les autres candidatures. Néanmoins, certains reconnaissent qu'ils sont souvent amenés, dans l'urgence, à recruter des personnes immédiatement opérationnelles. Dès lors, leur choix se porte sur des candidats censés connaître le public dont ils auront la charge. De ce point de vue, les critères mis en avant par les responsables de recrutement obéissent à une double logique. D'une part, celle qui consiste à exiger des candidats des compétences à la fois scolaires et professionnelles, susceptibles de répondre aux besoins et aux attentes de la profession : capacité de gérer un public en difficulté, de concevoir conjointement un projet social ou professionnel, de l'encadrer et de l'accompagner jusqu'au bout de ce projet; d'autre part, une logique qui serait davantage dictée par les impératifs et les conditions particulières de la mise en place des dispositifs sociaux et de leur réalisation.

Comme nous l'avons souligné, les milieux modestes, et le plus souvent issus de l'immigration, correspondent à la définition sociale et territoriale des publics cibles de ces dispositifs d'accompagnement social et professionnel (voir encadré 2).

La connaissance de ces populations constitue de plus en plus un impératif dans le bon déroulement des interventions menées auprès d'elles. Ainsi par exemple, la prise en charge de la diversité culturelle, depuis quelques années inscrite dans la formation des travailleurs sociaux, renforce l'exigence de trouver rapidement des personnes compétentes et familières des populations concernées par les dispositifs sociaux. Des enseignements en sociologie et en anthropologie, notamment sur les rapports interculturels, sont dispensés aux futurs travailleurs sociaux. Sous cette appellation, on retrouve des enseignements sur la sociologie de l'immigration, les relations interethniques, l'identité culturelle des descendants de migrants, les processus d'acculturation des migrants et de leurs enfants, les pratiques éducatives. Ils visent à mieux faire connaître ces populations aux futurs travailleurs sociaux. Il s'agit en outre de leur faire acquérir une compétence leur permettant de mieux appréhender les modes de fonctionnement de 
ces populations et d'être capable d'établir des relations d'écoute et d'échange avec elles. Ainsi, ils seront plus à même d'analyser et de gérer les situations d'interaction, voire de confrontation culturelle, et d'appliquer les solutions les mieux adaptées (Sainte-Mary Fly, 1997 ; Beski C., 1997). Il y a donc bien une singularité à travailler au contact de ces populations, mêmes lorsqu'elles sont nées et ont grandi dans l'hexagone ou qu'elles sont installées en France depuis plus de quarante ans. Dans ces conditions, le choix de professionnels sociaux descendants de migrants répond également à la nécessité de recruter des interlocuteurs capables de gérer les relations avec les publics dont ils ont la charge sans qu'ils aient besoin d'une formation spécifique.

Cette présomption de compétence s'observe concrètement dans le cadre de leur travail, et plus particulièrement dans les missions qui sont confiées aux conseillers à l'insertion. Plusieurs conseillères interviewées se voient très souvent confier les dossiers de jeunes filles en rupture scolaire ou familiale parce qu'on les juge plus aptes à comprendre les difficultés particulières auxquelles ces adolescentes sont confrontées au sein de leur famille. Cela suppose notamment que la proximité culturelle entre les conseillères et le public concerné participe à une meilleure prise en charge du projet social et professionnel de ce public. Cette dimension se vérifie également dans la répartition des tâches et des missions à caractère administratif ou des actions plus ponctuelles destinées à réguler les relations avec les jeunes. Par exemple, les dossiers concernant la citoyenneté et la discrimination seront plus facilement attribués aux professionnels descendants de migrants. Ces derniers interviennent également dans les situations conflictuelles opposant les jeunes qui fréquentent les Missions locales ou les Maisons de l'emploi et les autres professionnels travaillant dans ces structures. On les sollicite souvent pour tenter de résoudre ces conflits ou pour éviter les débordements possibles. Leur connaissance des milieux et les rapports souvent fraternels ou amicaux qu'ils entretiennent avec les jeunes les posent en conciliateurs ou en médiateurs, même si cela ne fait pas partie de leurs fonctions. Ils deviennent ainsi des vecteurs de régulation et de pacification des rapports avec les habitants et les publics concernés par les dispositifs sociaux.

La proximité réelle ou supposée avec les publics joue certes un rôle important dans la prise en charge et l'accompagnement des individus en quête de projet de vie ; cependant, elle soulève aussi une question de fond qui consiste à considérer les descendants de migrants comme une entité homogène et opaque, alors qu'ils constituent souvent un groupe très hétérogène. L'ancienneté de la migration des parents, leur pays d'origine, leur région d'installation, leur profession, leur rapport à la société française, sont autant d'éléments qui peuvent conduire les enfants de migrants à connaître des parcours et des expériences différentes. ( $c f$. par exemple M. Belhadj (2006) pour les Algériens). Des jeunes femmes m'ont affirmé qu'elles n'avaient jamais connu les « cités » car leurs parents, arrivés en France au début des années 50, ont toujours habité les quartiers des centre-villes. Elles n'ont donc jamais été confrontés au public qu'elles prennent en charge dans leur travail de conseillères. La connaissance d'un public en difficulté en général ou la connaissance de ce public en particulier, c'est-àdire les habitants de la zone ou du quartier défini socialement et géographiquement, voire ethniquement, reste une notion très relative. Tout comme la proximité sociale ou culturelle que l'on suppose entre tous les enfants de migrants dès lors que leurs parents sont d'origine maghrébine. Si elle n'est pas suffisante, cette proximité, réelle ou supposée, qui permet un rapport plus aisé avec le public concerné, joue un rôle important dans le choix des candidats.

Pour autant, la reconnaissance de ce type de compétence constitue t-elle toujours un atout? Elle semble parfois poser un problème d'éthique et de déontologie à certains responsables de recrutement ; ces derniers se défendent contre toute catégorisation visant à privilégier des critères personnels autres que ceux exigés par leur fonction.

\section{L'éthique professionnelle à l'épreuve des faits}

Les propos tenus par certains responsables de structures, chargés avec d'autres de recruter les candidats aux postes de conseillers ou de chefs de projet, révèlent un paradoxe et un décalage entre les attentes et les besoins souvent urgents auxquels ils sont confrontés et les valeurs ou principes qu'ils souhaiteraient voir appliquer dans leurs pratiques professionnelles. On recherche un candidat parce qu'il remplit les conditions de diplôme et d'expérience exigées 
pour le poste et non parce qu'il détiendrait un savoir spécifique lié à sa proximité avec les publics concernés. Il est parfois difficile de déceler dans cette affirmation de principe la part de rationalité qui ne supporterait aucune concession et celle qui est imposée par la réalité objective et les conditions d'exercice. Dans les faits, les candidatures reçues émanent essentiellement d'enfants de migrants ou d'émigrés venus poursuivre des études en France. Au-delà de toutes considérations personnelles, il y a une plus forte probabilité pour que la sélection s'opère à l'intérieur de cette catégorie de la population. Ce qui peut poser quelques problèmes à certains directeurs de structure soucieux de maintenir un équilibre dans la constitution du personnel et dans le rapport qui pourrait s'établir avec le public fréquentant les Missions locales ou les Maisons de l'emploi. L'un des responsables nous a confié à demi-mots qu'il serait plus enclin à choisir un candidat n'ayant pas a priori de proximité avec les jeunes fréquentant la Mission locale ni avec leur environnement social. Ce qui confirme la tendance constatée plus haut de l'orientation massive des diplômés descendants de migrants vers le secteur social, et en particulier vers l'intervention sociale :

«Il faut prendre de la distance par rapport à son propre vécu et quelqu'un qui aurait une histoire qui ressemble à celle des jeunes que l'on reçoit ou qui confond sa trajectoire avec celle du public, ce n'est pas du tout évident. »

(Alain, 45 ans, directeur d'une Mission locale, chargé de recrutement, titulaire d'un diplôme d'État d'assistant en service sociale, longue expérience dans le travail social et au sein des Missions locales).

Selon ces responsables, une trop forte proximite est susceptible de créer des liens trop intimes, trop fraternels avec les jeunes et deviendrait difficile à gérer par la suite. Elle aboutirait alors à une confusion des rôles et réduirait l'efficacité du travail des professionnels. La distance est considérée comme plus constructive qu'une trop grande proximité car elle facilite le travail du conseiller. Le recrutement d'une jeune femme sur ces critères s'est pourtant soldé par un échec; la personne est en effet partie au bout de deux mois ; il en a été de même d'un jeune homme habitant le quartier et qui a été jugé trop proche des jeunes. Une trop grande distance et une forte proximité semblent produire les mêmes effets selon certains responsables de recrutement: dans un cas, on méconnaît les conditions d'existence des jeunes, et on peine à évaluer la situation, et dans l'autre «on ne les connaît que trop " et on a donc tendance à manquer d'objectivité et à surestimer les difficultés rencontrées par les jeunes. Dans ce cas, la proximité avec le public est perçue comme un obstacle au bon déroulement des missions. Pourtant, lorsqu'on confronte ces propos à ceux des personnes recrutées par ces mêmes responsables, un décalage apparaît qui confirme la contradiction observée dans certaines attitudes de directeurs de structure. La plupart des professionnels interviewés dans le cadre de notre enquête et qui travaillent dans ce secteur avouent qu'ils sont là aussi (mais pas seulement) parce qu'ils sont fils ou filles de migrants et donc supposés mieux à même de faire face au public concerné et aux missions confiées :

« [...] Il n'y pas que la formation et l'expérience qui intéressent les recruteurs, il y a aussi notre origine maghrébine. Qu'on ait habité dans les quartiers et qu'on soit d'origine maghrébine c'est un plus. Même s'ils ne le disent pas ouvertement. On est censé connaître les quartiers dans lesquels on travaille et le public qui vient nous voir. On a un vécu en plus d'avoir fait des études supérieures et d'avoir déjà travaillé dans le social. » (Sélim, 33 ans, conseiller en insertion dans une Mission locale, maîtrise d'anthropologie)

Le décalage entre les propos des professionnels et des responsables de recrutement résulte sans doute d'une volonté institutionnelle d'ouvrir ces postes à un large public, tout en insistant sur la compétence professionnelle et l'efficacité des personnes recrutées. Les motifs évoqués semblent également fournir aux responsables de recrutement un argument objectif pour légitimer leur choix, indépendamment des savoirs et des connaissances particulières que les personnes recrutées détiennent ou sont censées détenir.

\section{Un recrutement le plus ouvert possible}

Certains directeurs de structures, souvent immigrés venus poursuivre leurs études supérieures en France, apparaissent plus enclins que leurs collègues à refuser tout particularisme des candidats. Ils optent d'emblée pour un recrutement le plus ouvert possible, quitte à écarter les candidatures des enfants de migrants. Les arguments de la surreprésentation et de la nécessaire diversification reviennent sans cesse dans leurs propos. Alors qu'eux-mêmes ont «bénéficié » de 
cette catégorisation, ils souhaitent la remettre en cause en invoquant leur attachement aux principes égalitaires et universels. Quête d'universalisme ou crainte d'être accusés de favoritisme?

Ils sont peu nombreux dans ce champ d'activité ; leur attitude est donc intéressante à plus d'un titre. Elle révèle toute l'ambiguïté de cette logique de recrutement et de sa conception de l'égalité. Tout en reconnaissant certaines compétences spécifiques, on s'efforce de maintenir un équilibre dans l'accès aux emplois sociaux entre les candidats descendants de migrants et les autres. Le dosage s'opère par le biais de consignes qui émanent le plus souvent d'élus locaux chargés de l'insertion et qui recommandent aux responsables de structures ou d'équipes de respecter cet équilibre. À cet égard, les propos qui nous ont été rapportés par certains professionnels, candidats à un poste de conseiller dans une Mission locale, sont très explicites. Une des directrices de cette structure a clairement spécifié à l'une de ses collègues qu'elle n'accepterait plus de candidatures de fils ou filles de migrants car ils étaient suffisamment nombreux au sein de l'établissement. Ce qui apparaît diffus dans les attitudes des autres responsables de structure est plus manifeste parmi les responsables de structure, originaires du Maghreb; il existe en effet des formes de pressions exercées par les élus locaux pour diversifier le personnel. Réduire ou augmenter le nombre de personnes appartenant à telle ou telle catégorie, sous prétexte que celle-ci est trop nombreuse ou pas suffisamment représentée dans un secteur donné, reviendrait à instituer des quotas spécifiques en fonction des appartenances sociales ou ethniques des individus et par conséquent à les stigmatiser encore davantage. Cette problématique révèle en fait les limites et les effets pervers que peuvent générer certaines logiques de catégorisation, quels que soient les critères sur lesquels elles se fondent. Ces logiques peuvent se retourner contre ceux qui en font l'objet et produire des effets inverses à ceux escomptés. Affirmer les principes que l'on considère comme fondamentaux dans l'exercice d'une profession participe certes à l'élaboration de l'éthique et de l'identité professionnelle, à condition de clarifier les cadres de cette élaboration pour éviter toute contradiction pouvant être à l'origine d'autres formes de discrimination.

Pour autant, la position de ces responsable de recrutement ne semble pas être partagée par certains professionnels interviewés qui considèrent qu'ils sont au contraire peu nombreux dans ce domaine d'activité, comme en témoigne Karim, responsable d'une Régie de quartier et très impliqué au sein du quartier dans lequel il travail :

"Je trouve qu'on n'est pas assez dans ce type de structure à des postes importants. On se retrouve le plus souvent sur des postes de chargés de suivi social, d'encadrement mais jamais à un niveau de responsabilité. On essaie dans les réunions départementales de leur montrer ce qu'on met en place sur les quartiers Nord. On est les seuls d'origine maghrébine, ce qui ne gâche rien. On apporte des idées, des concepts et on essaie d'aider les autres. En sachant qu'on a un avantage, on a une connaissance des quartiers. Je suis moi-même issu des quartiers, on arrive à discerner le bon du mauvais et le bon est plus fréquent qu'on le croit...» (Karim, 36 ans, directeur d'une Régie de quartier, titulaire d'un diplôme supérieur d'expert-comptable)

En reconnaissant qu'ils sont les mieux disposés pour remplir certaines fonctions, ils s'inscrivent d'une certaine manière dans cette logique catégorielle que nous avons décrite plus haut et qui tend à privilégier, pour des raisons à la fois objectives et subjectives, les profils de candidats descendants de migrants. Cependant, ils attribuent un sens différent à cette logique puisqu'ils se réfèrent davantage aux projets et aux objectifs qu'ils souhaitent réaliser dans le cadre de leur travail, en termes non seulement de valorisation des compétences et de mobilité sociale mais aussi de renouvellement des pratiques professionnelles.

\section{RENOUVELER LES RAPPORTS SOCIAUX}

La logique catégorielle que nous venons de décrire se confronte à la volonté des professionnels rencontrés de travailler dans ce secteur d'activité, même lorsque leur formation et leurs compétences ne les y préparent pas. Ils participent ainsi à leur propre catégorisation. Quel sens donnent-ils à cette participation et quelles en sont les répercussions, tant pour les professionnels sociaux en termes de parcours et de projets, que pour le secteur du développement social en termes de renouvellement des conceptions et des pratiques professionnelles? Plus que les emplois occupés ou les statuts qui restent à définir ou à redéfinir, ce sont surtout les objectifs assignés dans le 
cadre de leurs missions (comme le suivi et l'accompagnement du public auquel ils sont confrontés, les projets qu'il tentent d'élaborer et de concrétiser conjointement, soit par le biais d'une insertion professionnelle soit par le maintien d'un lien social fragilisé après la perte d'un emploi ou d'un logement), qui semblent le plus motiver leurs actions. De ce point de vue, on distingue deux types de rapport au travail qui diffèrent en fonction à la fois des parcours des professionnels et de leurs conceptions, de leur rôle et de leur degré d'implication et d'investissement dans leur travail. On distinguera, d'une part, une conception du travail social fondée sur la solidarité et sur un engagement social et, d'autre part, une conception plus contractuelle mais très personnalisée et diversifiée de l'accompagnement des individus et de leur projet. Mais au travers de ces deux attitudes, les motivations des professionnels traduisent une même volonté, celle d'exercer leurs fonctions différemment à travers une approche très personnalisée du travail social. À partir de ces deux types de rapport au travail et des objectifs que les professionnels sociaux s'assignent dans le cadre de leur mission, nous tenterons donc de rendre compte du rôle qu'ils jouent dans le processus de renouvellement des cadres légaux d'exercice et des rapports sociaux et professionnels.

\section{Une conception du travail social fondée sur l'engagement et la solidarité}

Le premier type de rapport se caractérise par un investissement professionnel fort et un ancrage important dans l'espace social. Il concerne essentiellement des responsables de projets et de structures d'insertion professionnelle (Régies de quartier) qui sont aussi les plus confrontés aux difficultés des habitants. Très impliqués dans leur travail, ils se posent en acteurs et en vecteurs d'un projet collectif. Leur parcours de militants ou de responsables d'association locales les a conduits à s'engager sur le terrain et auprès des élus locaux pour faire entendre leur voix sur les missions à mener au sein du quartier. L'un d'entre eux nous a confié que sa plus grande satisfaction était de pouvoir procurer une activité aux personnes socialement fragilisées afin de les aider à retrouver leur dignité. Au nom de ce même engagement, il s'est installé au cœur de ce quartier populaire de la région parisienne après des études d'expertcomptable. A priori, sa formation ne l'a pas préparé à diriger une structure d'insertion, ni à travailler dans le social. Le passage s'est opéré par le biais de la création d'une entreprise d'insertion dans le cadre du contrat emploi-ville, entreprise spécialisée dans l'entretien des espaces verts. Cette expérience a duré deux ans, le temps de se familiariser avec les dispositifs existants et de se faire connaitre dans les milieux professionnels et institutionnels : mairie, préfecture, conseil général. On lui demande alors de restructurer une Régie (voir encadré 3) située dans la commune voisine. Des désaccords avec les politiques de la Ville menées par la municipalité le conduisent à accepter les propositions des élus locaux et à s'installer dans

\section{Encadré 3 \\ La mission et les objectifs d'une Régie de quartier}

Une Régie de quartier regroupe les collectivités locales, les logeurs sociaux et les habitants du ou des quartiers qui composent son territoire. Ensemble, ils interviennent sur la gestion urbaine de cet espace.

Sa mission technique est d'entretenir, d'embellir et de veiller sur le quartier. Cela se traduit par une activité qui favorise l'insertion sociale et professionnelle d'habitants en difficulté et l'émergence de nouveaux services. Les activités de la Régie sont le support d'une démarche de médiation et de sensibilisation qui poursuit une mission sociale auprès des habitants et des acteurs locaux: améliorer les relations humaines, inventer des modes de gestion urbaine partagée, et surtout construire une citoyenneté active sur le territoire de la Régie en suscitant l'implication et la responsabilisation des habitants. La Régie de quartier est un outil de gestion territorialisé d'un quartier qui vise à répondre aux besoins spécifiques de la cité. Elle vise aussi à développer des liens entre les habitants et à favoriser le développement économique et l'insertion sociale et professionnelle dans la zone concernée. Le public visé est majoritairement composé de personnes en difficulté ou en situation précaire habitant le quartier. 
une autre commune où il développera deux structures : une Régie dans les quartiers Nord de la ville et une structure d'insertion dans le recyclage informatique qui comporte environ douze postes en insertion à temps complet. En outre, il est chargé, avec d'autres associations locales, des animations du quartier au travers notamment de campagnes d'informations sur des questions touchant à la citoyenneté ou à la discrimination ou encore par le biais d'activités ludiques et festives autour de l'organisation de fêtes de quartier.

Changer l'image des quartiers est aussi une tâche que se sont assignée les responsables des Régies de quartiers et les associatifs ; à cet effet, ils se mobilisent à la fois sur le terrain et dans les actions menées en direction des conseils régionaux et de la préfecture. Ils doivent tout à la fois agir sur les difficultés rencontrées par les habitants et sur l'environnement social en tentant de réhabiliter l'image de ces quartiers longtemps délaissés et stigmatisés :

"Il n'y a pas que du mauvais dans les quartiers, il y a beaucoup de choses positives mais on ne sait pas les mettre en évidence. Ce sont des choses que j'essaie modestement de faire comprendre au niveau de la préfecture. Il faut montrer ce qu'il y a de bon dans les quartiers et pas seulement la violence ou la drogue. Il faut arrêter de stigmatiser. Vous êtes venue tout à l'heure, vous n'avez pas été agressée ? ... » (Farid, 40 ans, chef de projet contrat de ville, titulaire d'un diplôme d'éducateur spécialisé).

Leur discours montre que la mission qu'ils se sont fixée dans ce projet va au-delà de l'objectif principal qui consiste à procurer une activité aux personnes en difficulté ; cette mission englobe en effet l'intégration de tout un environnement dans le tissu social et dans la vie de la cité. Cela implique d'une part, une proximité avec les habitants et leur quotidien et d'autre part, un réseau d'acteurs de la vie sociale et locale pour une meilleure prise en charge des personnes et de leur cadre de vie. Dans ce contexte, on comprend mieux que la personnalité même des professionnels, leur capacité à se faire connaître des habitants, à être acceptés par eux et à établir avec eux une relation de confiance et de respect mutuel sont des éléments fondamentaux dans la réussite de leur mission. Le fait d'être proche des habitants et de partager un même cadre de vie, d'être confronté aux mêmes difficultés, tout cela fait naître le sentiment que l'on appartient à une même communauté, à une même famille. On retrouve la notion de «commu- nauté » au sens wébérien du terme, c'est-à-dire un groupe d'hommes et de femmes qui, à un moment et à un endroit donnés, ont partagé un même destin à travers un mode de vie, des valeurs, une histoire et une expérience communes. Dans le cas qui nous occupe, cela renvoie à une solidarité à l'égard de ses semblables et des personnes les plus vulnérables en particulier. En ce sens, il ne s'agit pas d'un sentiment d'appartenance à une ethnie, même si parfois le groupe d'origine est invoqué, mais du partage d'un ensemble de valeurs humaines comme le respect, l'entraide, l'assistance, la solidarité, acquises au cours de la socialisation et que ravivent certains événements, comme le drame de la canicule de l'été 2003. C'est certainement l'exemple le plus cité par ces professionnels pour montrer encore une fois l'entraide et la solidarité qui existent ici plus qu'ailleurs :

"Il y a une vraie solidarité ici, un esprit de famille car on se connaît tous. Les 1000 morts de la canicule étaient presque tous des personnes âgées, c'est une chose que l'on ne verra jamais ici ni dans les pays maghrébins ou dans notre communauté, ici en France. Il y a une convivialité et un esprit différents, on ne laisse pas mourir les personnes âgées. " (Karim, 36 ans, responsable d'une Régie de quartier, titulaire d'un diplôme supérieur d'expert-comptable).

C'est ainsi qu'ils mobilisent tout un réseau d'acteurs de la vie locale et tout ce qui constitue leur quotidien afin de mieux prendre en charge les personnes et leur cadre de vie.

Un autre de leurs objectifs est de modifier les représentations et les rapports à l'œuvre dans le champ du travail social et qui, selon eux, nuisent aux relations de confiance et de respect mutuel indispensables entre les travailleurs sociaux et les habitants concernés par les dispositifs sociaux :

"Les travailleurs sociaux, et notamment les assistantes sociales, n'ont pas su établir des relations de confiance avec les familles immigrées. Ils avaient, et pour certains, ils ont encore, des représentations totalement fausses de la situation de ces familles. Ils n'ont jamais vraiment réussi à les comprendre en fonction de leur vécu, de leur transplantation dans un pays qu'elles ne connaissaient pas et où elles étaient obligées du jour au lendemain de s'adapter. Ils n'ont pas tenu compte de tout ça et du coup les relations qu'ils entretenaient avec ces familles étaient simplement des relations d'assistés et de dominants- 
dominés. Un fossé s'est creusé entre ces familles et les travailleurs sociaux, ce qui a rendu leur travail plus difficile et moins efficace. Moi je ne conçois pas mon travail comme ça. Les relations que j'entretiens avec les personnes qui viennent me voir sont équilibrées; elles me sollicitent pour telle ou telle chose et j'essaie d'y répondre en tenant compte de beaucoup d'éléments qui sont aussi constitutifs de la personne. Il faut considérer les gens comme des personnes à part entière avec leur vécu et leur histoire. " (Mourad, 38 ans, responsable de projet dans le cadre du contrat de Ville, DESS de gestion des entreprises)

Pour ces professionnels, il s'agit avant tout de changer le regard qui est souvent porté sur ces populations et d'instaurer avec elles d'autres types de relations moins hiérarchisées et plus équilibrées. Cela constitue une condition indispensable pour comprendre les situations souvent complexes auxquelles les travailleurs sociaux sont confrontés. Ne plus considérer uniquement les individus comme des assistés mais comme des individus à part entière, en intégrant leur histoire et leurs parcours dans le projet qui sera élaboré avec eux, contribue à davantage de proximité. Cette question est centrale car elle met en évidence les carences longtemps décriées dans le champ du travail social, carences notamment liées à la représentation des milieux populaires, et des émigrés en particulier, longtemps considérés par certains travailleurs sociaux comme « des personnes à problèmes cherchant dans un ailleurs ce qu'il ne peuvent trouver chez eux" (Ibid, 1993, p. 82). Dans cette vision, les milieux défavorisés et les immigrés les plus modestes, en particulier, ne sont jamais appréhendés comme des personnes autonomes, capables de prendre des décisions et de se prendre en charge pour changer leur situation (Ibid, pp. 82-91).

\section{Une approche personnalisée et diversifiée de l'accompagnement}

L'autre type de rapport caractérise essentiellement les conseillers en insertion qui travaillent la plupart du temps au sein des Missions locales. Ils ont en général un rapport plus pragmatique à leur travail que celui des autres professionnels interviewés (responsables de Régies de quartier, chefs de projet). Leur mission consiste principalement dans l'aide, l'accompagnement et le suivi de jeunes âgés de moins de 25 ans en rupture scolaire ou familiale. Leur statut de cadres ou d'ingénieurs les rend plus dépendants de la structure dans laquelle ils travaillent et moins autonomes dans les prises de décisions ; ce qui limite très souvent leur prise d'initiatives et la portée de la mission dans laquelle ils souhaiteraient s'engager davantage. Mais surtout, les parcours de ces professionnels sont différents de ceux des responsables de Régies de quartiers ; ils ont une formation universitaire en sociologie, psychologie ou en anthropologie, au sein de laquelle la question sociale occupe une place importante; par ailleurs, ils possèdent tous une expérience confirmée dans le travail social. Ils sont plus jeunes et moins engagés socialement et politiquement que leurs aînés (issus souvent des mouvements associatifs des années 80). Leurs parcours ressembleraient à ceux de tout professionnel du social s'ils ne travaillaient pas dans ce secteur particulier du développement social urbain et s'ils n'étaient pas descendants de migrants, censés détenir une compétence et un savoir endogènes nécessaires pour travailler dans ce secteur. De même, le fait de travailler dans des structures implantées un peu à l'écart des quartiers explique en partie la moindre proximité avec les habitants. Ces conseillers connaissent surtout les jeunes qui fréquentent les locaux des Missions locales ou des PAIO et c'est exclusivement avec eux qu'ils entretiennent des relations. Leur travail ne nécessite pas une proximité avec l'environnement social et avec ses habitants. Pour autant, cette dimension n'est pas absente de leur mission. Ils sont souvent amenés à nouer aussi des relations avec les familles des jeunes qu'ils encadrent et accompagnent professionnellement. Ils sont aussi en contact avec les autres professionnels travaillant au cœur des quartiers et qui connaissent suffisamment bien les habitants pour les aiguiller dans leurs actions. De telles conditions les conduisent très souvent à adopter une conception différente à l'égard de leur travail afin de trouver un équilibre dans leurs relations avec les jeunes.

Ils s'attachent ainsi davantage peut-être que les autres professionnels interviewés à maintenir une distance avec le public, tout en reconnaissant une certaine proximité avec lui. Leur objectif est précisément que cette proximité n'interfère pas directement dans leur travail afin d'établir des rapports équilibrés et de respect mutuel avec les jeunes. La question de l'éthique ou de la posture professionnelle se pose pour ces professionnels dans l'articulation entre une 
distance nécessaire et une proximité maîtrisée. Les observations effectuées au cours de l'enquête montrent que les hommes recrutés comme conseillers en insertion, tout comme les chefs de projets ou les responsables de Régies de quartier, ont en général des rapports fraternels avec les jeunes inscrits à la Mission locale. Cette proximité, plus ou moins souhaitée par les uns et revendiquée par les autres ("quand on connaît mieux les habitants on peut mieux les aider »), s'intègre dans leurs pratiques professionnelles comme un élément constructif.

Cette question se pose avec plus d'acuité pour les femmes conseillères en insertion qui s'efforcent de maintenir une plus grande distance avec les jeunes qu'elles sont chargées d'accompagner dans leur projet. Le vouvoiement, par exemple, est plus souvent utilisé, même si par ailleurs elles se sentent proches de ce public et des filles en particulier. Plus que les hommes, les conseillères sont confrontées à la question de l'autorité à l'égard des garçons, généralement moins disposés à accepter les recommandations émanant de la gente féminine. Elles s'efforcent donc de faire preuve de tact et de fermeté afin d'instaurer un climat de confiance et de respect mutuel :

«Ce n'est pas chose facile, mais on y arrive..., le plus impressionnant chez eux c'est leur taille, ils sont beaucoup plus grands que moi." (Sonia, 28 ans, conseillère en insertion dans une Mission locale, titulaire d'un DEA en sociologie)

La distance qu'elles tentent de maintenir dans leurs relations avec les jeunes est aussi pour elles un moyen d'éviter la confusion des rôles qui pourrait affecter la représentation que les jeunes ont de la conseillère (considérée comme la grande sœur par les filles et comme la femme d'origine maghrébine par certains garçons, avec tout ce que cela implique de clichés et de représentations traditionnelles). De ce point de vue, leur tâche n'est pas aisée mais elle est aussi essentielle; elles jouent en effet un rôle régulateur auprès des garçons en s'imposant à travers un statut qui instaure une vision et des rapports différents de ceux qui peuvent s'établir en dehors du cadre professionnel. Avec les jeunes filles, les relations sont souvent plus aisées. Leur proximité avec les conseillères qui appartiennent aux mêmes milieux fait souvent naître un sentiment de confiance qui facilite l'accompagnement et le suivi des projets. Leur connaissance du milieu familial et parfois le partage d'une expérience permet à ces jeunes conseillères de mieux appréhender les situations rencontrées dans leur complexité et leur diversité. La recherche de compromis entre les jeunes filles en quête de repères et la famille de ces dernières constitue pour ces professionnelles un élément essentiel dans le bon déroulement de leurs missions :

«Je suis souvent confrontée dans mon travail à faire plus de social que d'insertion professionnelle. Il y a des filles qui viennent me voir parce qu'elles ne s'entendent plus avec leurs parents et qu'elles veulent partir de chez elles alors qu'elles n'ont pas de boulot. Elles veulent que je leur trouve un hébergement et un travail. C'est pas simple, je leur explique que mon rôle c'est de les aider et de les accompagner dans leur projet professionnel et que ça c'était plus le rôle de l'assistante sociale. Mais en même temps je sais que les assistantes sociales ne sont pas bien formées pour s'occuper d'elles. Elles prennent des décisions parfois radicales et cela fait plus de mal que de bien. C'est vrai que moi j'essaie plutôt de trouver des solutions qui ne soient pas en rupture avec la famille. Je sais que même si elles sont en conflit avec elle, la famille reste un vecteur d'équilibre pour les filles et j'essaie de comprendre véritablement ce qui se passe pour savoir s'il n'y a pas moyen de trouver des compromis entre la fille et sa famille, c'est important même si ça outrepasse mes fonctions. ॥ (Karima, 32 ans, conseillère en insertion dans une Mission locale, DEA en anthropologie)

Ainsi, ces professionnels endossent parfois un rôle qui dépasse le cadre de leur mission. Cette démarche, qui s'inscrit aussi dans les attentes des recruteurs, semble surtout traduire le souci de mieux prendre en charge des situations qu'elles jugent mal comprises par certains travailleurs sociaux (Bourg, 1997). Le fait d'empiéter sur les prérogatives de leurs collègues est aussi guidée par une volonté de s'inscrire davantage dans une approche personnalisée de l'encadrement et de l'accompagnement des jeunes. Dans cette approche, les projets sont élaborés conjointement avec les individus, considérés à la fois dans leur individualité et dans leur parcours social et familial. C'est aussi pour cette raison que la famille sera le plus souvent possible associée au projet d'insertion du jeune, même lorsque celui-ci est en conflit ou en rupture avec elle. Ces professionnelles partagent, de ce point de vue, la volonté des chefs de projets et des responsables de Régies de quartier d'établir des relations différentes avec ces populations et de parvenir à 
une solution pacifiée et plus négociée des problèmes que rencontrent les filles, notamment au sein de leur environnement social ou familial.

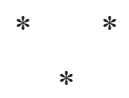

L'insertion sociale et professionnelle s'est imposée comme un enjeu capital ces dernières années; en transformant les bases mêmes du travail social, elle a représenté pour tous les professionnels du travail social un moyen de faire reconnaître les compétences objectives acquises au cours de leurs études mais aussi au cours de leurs différentes expériences (professionnelles et personnelles). La présomption de compétence dont les descendants de migrants font l'objet au sein du secteur du développement social est certes un critère important dans leur recrutement, mais elle n'est pas déterminante. Si les compétences endogènes (proximité sociale, culturelle avec les habitants et les publics concernés par les dispositifs sociaux) représentent un avantage pour beaucoup de responsables de recrutement, elles sont parfois remises en cause et deviennent un obstacle pour d'autres. Sans une solide formation universitaire et généraliste, les professionnels sociaux interviewés dans le cadre de notre enquête ne seraient certainement pas recrutés.

Ce n'est que lorsqu'ils réunissent toutes ces compétences qu'ils représentent les candidats «idéaux » pour les institutionnels. Pour autant, leur tâche ne sera pas aisée ; ils sont conscients du rôle qu'ils auront à remplir auprès des publics dont ils ont la charge. C'est donc en parfaite connaissance des règles et des conditions qui régissent le déroulement de leurs missions qu'ils ont choisi de travailler dans ce secteur particulier du travail social. En ce sens, leur professionnalisation n'est pas seulement le résultat d'une logique catégorielle des procédures de recrutement, basée à la fois sur la reconnaissance de savoirs scolaires et professionnels et sur une présomption de compétences endogènes. Elle est également le fruit d'une rationalisation de leur travail et de leur capacité à valoriser et à faire reconnaître leurs compétences au sein du champ du travail social. La place et le rôle de ces professionnels descendants de migrants constituent donc bien un enjeu qui dépasse le strict cadre du travail social. Leur conception du travail traduit une volonté accrue de modifier les représentations et les rapports avec les publics concernés par les dispositifs sociaux d'aide et d'accompagnement. Ils souhaitent établir des relations plus négociées et plus équilibrées entre les professionnels et les individus afin de gommer la hiérarchisation des relations dominantsdominés et de développer des rapports de confiance et de collaboration. Concevoir l'individu dans son histoire et l'intégrer dans un projet élaboré conjointement apparaît comme un impératif de leur fonction. Cette conception de leur travail, fondée sur un rapport de proximité avec les personnes, répond souvent à la volonté d'une prise en charge personnalisée et diversifiée des situations rencontrées. Une telle démarche ne déroge pas aux prérogatives professionnelles de ces nouveaux professionnels, elle est même inscrite dans les référentiels du travail social. Cependant, elle ne peut se mettre réellement et efficacement en place sans l'implication et l'investissement d'une majorité de professionnels qui, au regard de leurs propres expériences, semblent peut-être plus disposés que d'autres à modifier les cadres légaux et les rapports sociaux et professionnels.

Les apports de ces nouveaux professionnels, quelle que soit leur nature, ne peuvent être qu'utiles et constructifs à la fois pour le renouvellement de la profession et pour les acteurs eux-mêmes, investis d'une mission et d'un rôle qui dépassent parfois le cadre de leurs fonctions. Certains d'entre eux se posent même en interlocuteurs auprès des collectivités locales afin d'être reconnus non seulement pour ce qu'ils sont mais aussi pour ce qu'ils font. Cette reconnaissance passe aussi par la possibilité d'accéder aux postes à responsabilité au même titre que n'importe quel autre professionnel compétent et désireux de s'impliquer davantage dans la vie de la cité. À cet égard, quelles perspectives ce champ d'activité peut-il leur ouvrir afin de leur permettre de sortir du cadre strictement local et territorial pour se diriger vers des domaines plus larges et moins connotés socialement et géographiquement? Beaucoup ont évoqué cette question lors des entretiens. Pour les directeurs de Régies de quartiers ou les responsables de projets, il s'agit surtout d'accroître la portée de leurs actions, d'élargir leur champ d'activités en intégrant les instances générales ou régionales. Cela constituerait comme un prolongement de leurs engagements sociaux et politiques. Les autres professionnels voudraient quant à eux changer de secteur, créer leur propre entreprise, s'engager dans une carrière de journaliste ou de fonctionnaire dans les ministères. Le travail social apparaît donc comme une étape dans leur parcours 
professionnel susceptible de leur permettre d'accéder à des secteurs plus diversifiés.

Reste à connaître les conditions offertes à ces professionnels pour accéder à d'autres domaines d'activités et les moyens qu'ils mettent en œuvre pour y parvenir. La question de l'évolution de ces professionnels vers d'autres domaines d'activités est essentielle; en effet, l'issue qu'elle préfigure permettrait de montrer que les objectifs des politiques territo- riales destinées à réduire les inégalités ne résident pas uniquement dans une définition catégorielle mais dans la participation au processus d'intégration globale des individus. En permettant notamment aux professionnels qui le souhaitent de sortir du secteur du développement social pour enrichir leurs compétences au contact de milieux divers, les institutions pourront ainsi disposer de personnes compétentes dans un plus large éventail d'activités.

\section{Bibliographie}

Amselle J.-L. (1996), Vers un multicularisme français. L'empire de la coutume, Paris, Aubier.

Bataille P . (1997), Le racisme au travail, Paris, La Découverte.

Belhadj M. (1998), Une "révolution" tranquille, stratégies professionnelles et dynamique familiale d'un groupe de femmes françaises d'origine algérienne, Thèse de doctorat en sociologie, Paris EHESS.

Belhadj M. (2006), La conquête de l'autonomie, histoire et parcours d'une génération de femmes françaises descendantes de migrants algériens, Paris, Éditions de l'Atelier, Paris.

Beski C. (1997), «L'interculturel dans le travail social », Les Cahiers de l'Actif $\mathrm{n}^{\circ} 250-251$, marsavril.

Billion P. (2001), «Travail social et immigration, Traitement de la " différence " et territorialisation des politiques publiques », Les Cahiers du Cériem, n 8 décembre.

Billion P. (2004), «Les travailleurs sociaux dits “issus de l'immigration" ", Informations sociales $\mathrm{n}^{\circ} 113$, janvier, pp. 112-121.

Bourg D. (1997), « Travail social et intégration des jeunes filles issues de l'immigration maghrébine », Les Cahiers de l'Actif, $\mathrm{n}^{\circ}$ 250-251, mars-avril.
Chopart J.-N. (2000) (dir.), Les mutations du travail social, Paris, Dunod.

Chopart J.-N. (1994), " Observer les emplois et les qualifications des professions de l'intervention sociale », Mire Info, $\mathrm{n}^{\circ}$ 30, p. 52.

Cohen-Emerique M. (1993), « L'approche interculturelle dans le processus d'aide », Revue française de sciences sociales, $\mathrm{n}^{\circ} 171,4^{\mathrm{e}}$ trimestre.

Danzelot J. (1984), L'invention du social, Paris, Le Seuil.

Doytcheva M. (2005), Le multiculturalisme, Paris, La Découverte, collection « Repères ».

Hugues E.C. (1958), Men an their work, Glencoe, The Free press.

Ion J., Tricart J.-P. (1998), Les travailleurs sociaux, Paris, La Découverte.

Las P. (1990), «Travail social et immigration», Migrants-Formation, $\mathrm{n}^{\circ} 80$, mars.

Marticelli D., Wievorka M. (1992), «La crise du travail social : étendue et limites », Migrants-Formation, $\mathrm{n}^{\circ} 88$, mars.

Martiniello M. (1995), L'ethnicité dans les sciences sociales, Paris, PUF, « Que-sais-je ». 
Paradeise C. (1987), « Des savoirs aux compétences : qualification et régulation des marchés du travail », Sociologie du travail, $\mathrm{n}^{\circ}$ 1, pp. 35-46.

Paradeise C. (1987), «Acteurs et institutions. La dynamique des marchés », Sociologie du Travail $n^{\circ} 1$, pp. 79-105.

Schnapper D. (1994), La communauté des citoyens, sur l'idée moderne de la nation, Paris, Gallimard.

Schnapper D. (2002), La démocratie providentielle, Essai sur l'égalité contemporaine, Paris, Gallimard.
Vrancken D. (1993), L'hôpital et la question gériatrique. L'organisation hospitalière face au défi du vieillissement de la population, Paris, Fondation Nationale des Sciences Politiques, thèse de doctorat de l'Institut d'Études Politiques de Paris.

Vrancken D. (1994), « De la profession au marché du travail », in De Coster M., Pichault F., Traité de sociologie du travail, Bruxelles, pp. 253-273.

Weber M. (1995), Économie et Société, Paris, Plon, collection «Les classiques Agora», (1 ère édition 1920).

\title{
Résumé
}

\section{Quelle professionnalisation dans le travail social pour les diplômés issus de l'immigration?}

\author{
Marnia Belhadj
}

La mise en place des politiques de la Ville a profondément transformé les cadres d'exercice des professions sociales avec l'émergence de nouveaux emplois et de nouveaux acteurs dans le champ du travail social. Leur travail s'organise autour de l'aide, de l'intervention, de la prévention et de l'accompagnement des populations fragilisées habitant une zone définie socialement et territorialement. Ces fonctions mobilisent des compétences ou des savoirs particuliers : connaissance du milieu et de l'environnement social et territorial concernés par le dispositif. Beaucoup d'enfants de migrants diplômés de l'enseignement supérieur, issus eux-mêmes de quartiers populaires et désireux de faire reconnaître leurs compétences, ont trouvé là un moyen de concilier leur formation universitaire et leurs aspirations professionnelles. À partir d'une enquête menée dans deux communes de la région parisienne, l'article s'attache à analyser le rôle de ces nouveaux acteurs dans le champ social et dans le renouvellement des cadres et des rapports sociaux et professionnels.

\section{Mots clés}

Travailleur social, professionnalisation, population d'origine étrangère, enquête, lle-de-France. Journal of Economic Literature : J 15, J 71 